\title{
Phonon reflection at a sapphire-vacuum interface $\dagger$
}

\author{
Peter Taborek and David Goodstein \\ California Institute of Technology, Pasadena, California, 91125, USA
}

Received 1 February 1979

\begin{abstract}
Theoretical and experimental studies have been conducted of the transmission of phonons in sapphire and their reflection at a crystal-vacuum interface. The effects of crystal anisotropy on the reflection process, not previously noted in the literature, are discussed in detail. The heat-pulse technique has been used to obtain high-resolution timeof-flight spectra as a function of the angle of incidence in accurately known crystallographic directions. The agreement with predictions from numerical calculations is excellent. The relevance of these observations to studies of the anomalous transmission of energy through a crystal-liquid helium interface is also discussed.
\end{abstract}

\section{Introduction}

In recent years, a number of experiments have been reported in which phonons are generated, transmitted ballistically through a crystal and detected (Maris 1970, Taylor et al 1970). The generator and detector may be on opposite faces of the crystal (these are called transmission experiments) or on the same face, so that the detected phonons first reflect from the opposite surface (reflection experiments). This latter class of experiments in particular has been used to study aspects of the Kapitza resistance (Guo and Maris 1972, Long et al 1973, Kinder and Dietsche 1974, Horstman and Wolter 1977, Folinsbee and Harrison 1978, Weber et al 1978).

A spectrum typical of those observed in reflection experiments is shown in figure 1. For this and all other spectra presented in this paper, the generator is a $100 \mathrm{~nm}$ evaporated aluminium film used as an Ohmic heater and the detector is a $200 \mathrm{~nm}$ tin film operated as a superconducting bolometer with the help of a magnetic field. The crystal is sapphire, mounted so that it is immersed in liquid helium at $2.0 \mathrm{~K}$ except for the reflecting face, which is in vacuum. The spectrum is a record of signal intensity at the detector against time of flight after pulsing the heater. Three main peaks can be seen, attributable to the arrival of longitudinal phonons (the first to arrive), transverse phonons (the last) and phonons that have been mode converted upon reflection (in between). The sharp peaks arising from the reflection of ballistic phonons often lie on a broad background due to phonons which have scattered from bulk impurities.

Figure 2 shows a spectrum which is qualitatively different from those reported as typical. Here, six sharp peaks and some broader features can be clearly seen. In other orientations we have observed as many as eight sharp peaks. Our analysis of the processes of propagation and reflection of sound in real crystals indicates that there are as many as nine channels with different times of flight for transmitting energy from the heater to

† Supported in part by NSF Grant No. DMR77-00036 and a grant from the Caltech President's Fund. 


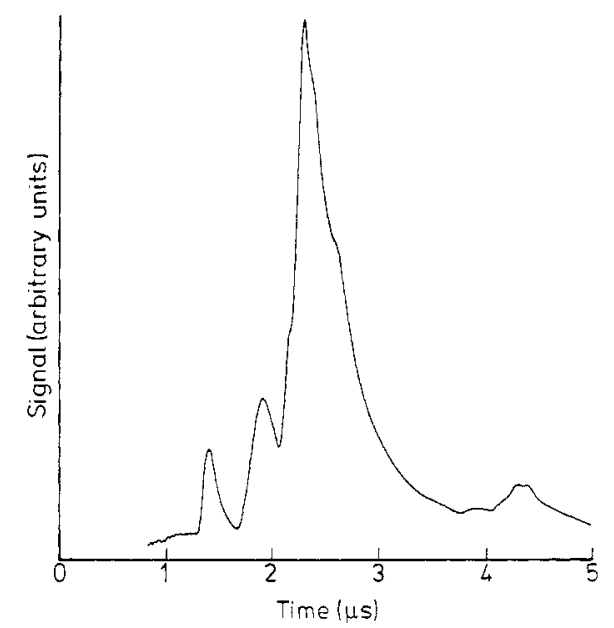

Figure 1. Low-resolution spectrum similar to those seen in the literature, showing longitudinal, mode-conversion and transverse peaks. The heater and bolometer were linear strips, $14 \mathrm{~mm}$ long, separated by $2.5 \mathrm{~mm}$. The sapphire crystal was $6.4 \mathrm{~mm}$ thick $\times 57 \mathrm{~mm}$ in diameter.

the bolometer via reflection from a flat surface. The purpose of this paper is to describe that analysis and to present experimental data which support the conclusions.

The principle new contribution of this analysis is concerned with the effect of crystal anisotropy on the reflected signal. It is well known that crystal anisotropy gives rise to a phenomenon known as phonon focusing. In any crystal, a packet of phonons with isotropically distributed wavevectors may have a very anisotropic pattern of energy flow. The phenomenon has been systematically investigated and its effect on phonon transmission has been widely noted (Maris 1970, Taylor et al 1970). The fact that the anisotropy of the elastic constants can dramatically affect the allowed reflection processes, as well as the directions and polarisations with which phonon beams are reflected, does not, however, appear to be so well understood.

We are concerned in this report only with the effects of specular reflection from an ideal flat surface. The basic condition governing specular reflection is that the component of the incident wavevector parallel to the surface is conserved in the outgoing waves.

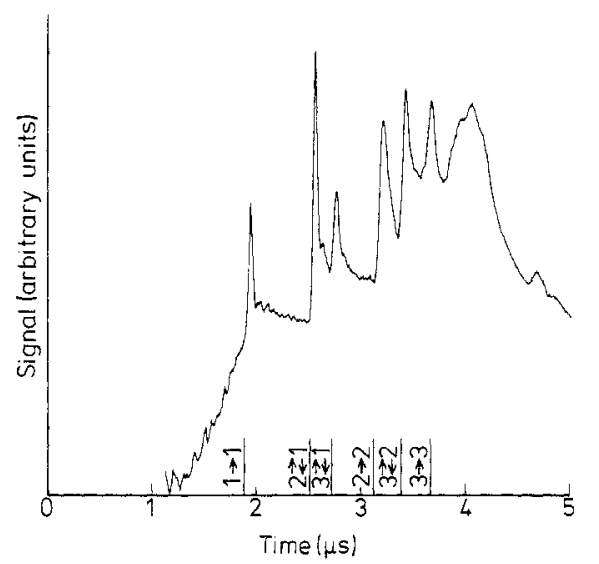

Figure 2. High-resolution spectrum showing six narrow peaks followed by a broad jump. The predicted arrival times of various channels, as discussed in $\S 3$, are shown on the abcissa. 
However, because of crystal anisotropy, the direction of energy flow (that is, of the group velocity) is not necessarily the same as that of the wavevector (or phase velocity). Although the law governing reflection is conveniently expressed in terms of $k$ vectors, the observed signal is due to energy flow from the generator to detector along a path defined by the group velocity vectors. This path is not simply related to the incident $k$ vector and is generally not coplanar with the $k$ vectors or the surface normal. Because the relationship between $k$ vectors and group velocity vectors is rather complicated, the prediction of the observed reflection signal is necessarily an iterative process. An incident $k$ vector must be guessed, the reflected $k$ vector and the path of energy flow computed and then the incident $k$ vector corrected until a path is found that carries energy from the generator to the detector.

In $\S 2$ of this paper we describe the analysis necessary to predict the times of arrival of the various reflected modes of our bolometer. Experiments designed to test the results of the analysis are present in $\S 3$ and the conclusions are discussed in $\S 4$.

\section{Analysis}

Let us first consider the predictions of isotropic elastic theory (Landau and Lifshitz 1970). An isotropic solid has two elastic constants which can be chosen to be the longitudinal and transverse sound velocities $c_{l}$ and $c_{\mathrm{t}}$. The two degenerate tranvserse sound modes can have any polarisation normal to the direction of propagation. To analyse

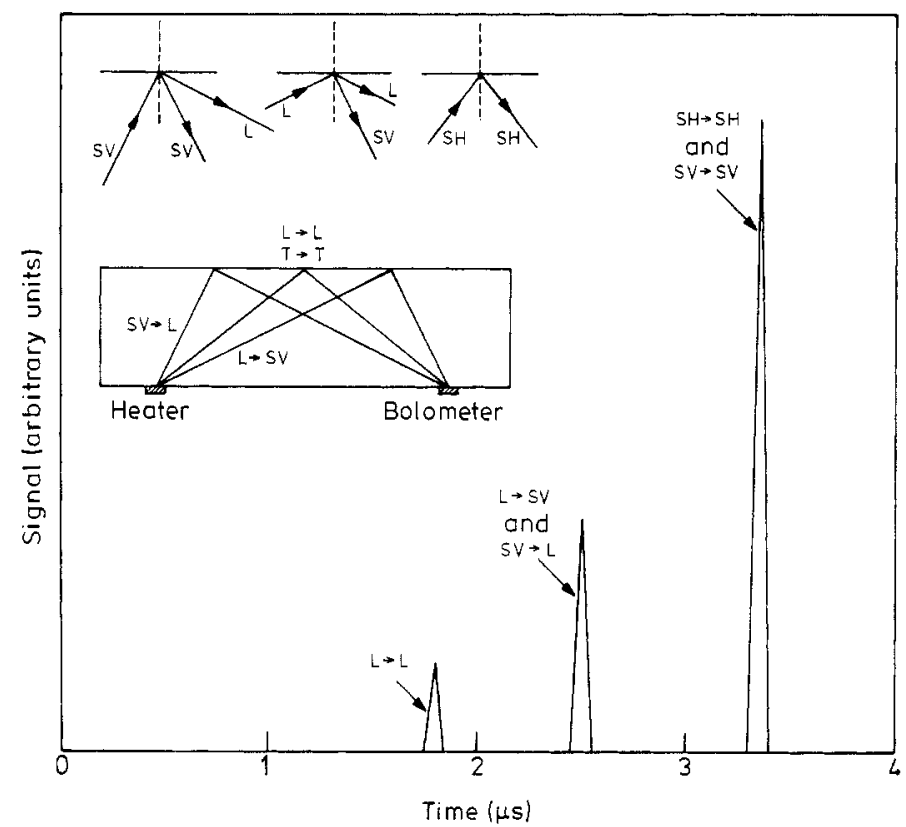

Figure 3. Schematic diagram of a bolometer signal assuming an isotropic solid similar to sapphire with sound speeds $c_{l}=1.11 \times 10^{6} \mathrm{~cm} \mathrm{~s}^{-1}, c_{t}=0.604 \times 10^{6} \mathrm{~cm} \mathrm{~s}^{-1}$. The heaterbolometer separation is $6.7 \mathrm{~mm}$, which is the same as was used for the spectrum in figure 6 . The calculation also inciudes the effects of a Debye density of states which accounts for the factor of approximately eight difference in the longitudinal $\rightarrow$ longitudinal and transverse $\rightarrow$ transverse peak heights. The reflection processes which contribute to each peak are also indicated. The inset shows the possible reflection processes and the consequent paths that connect the heater and the bolometer in an isotropic solid. 
reflection it is convenient to choose one transverse polarisation vector normal to the plane of incidence and the other in the plane of incidence. These are referred to respectively as SH and SV waves, SH standing for shear horizontal and sv for shear vertical (the terminology comes from the seismological literature).

The inset of figure 3 shows the paths that would connect the heater and bolometer in a reflection experiment. In addition to the non-mode-converted path for which the angle of incidence equals the angle of reflection, there are two mode-conversion paths. Although these have the same time of flight in an isotropic system, they are not entirely equivalent. The $\mathrm{sv} \rightarrow \mathrm{L}$ (longitudinal) path should dominate because more $\mathrm{T}$ (transverse) phonons are excited by the heater, because of their larger density of states at low frequencies. In addition, the two paths have different angles of incidence.

At normal incidence, all three modes are uncoupled and reflect independently. The SH waves are uncoupled from the other modes at all angles of incidence, but the SV waves convert to a longitudinal path with an amplitude that increases from zero at normal incidence up to a critical angle $\theta_{\mathrm{c}}=\sin ^{-1}\left(c_{\mathrm{t}} / c_{1}\right)$, where the reflected modeconversion amplitude drops back to zero.

The results of detailed calculations of signal intensity against time of arrival, including a Debye phonon density of states and solid-angle effects, are shown in figure 3. Note that signals corresponding to several different angles of incidence arrive at the bolometer even for an isotropic solid. Although it might be expected that this analysis, which predicts only three peaks, would be sufficient for sapphire, the most nearly isotropic of the commonly used crystals (e.g. the transverse sound speeds differ by only a few per cent), a comparison of figure 3 with figure 2 shows that a more sophisticated analysis is needed.

The starting point for the analysis of elastic waves in an anisotropic crystal is Newton's law in the form

$$
\rho\left(\partial^{2} u_{i} / \partial t^{2}\right)=\partial \sigma_{i k} / \partial x_{k}
$$

where $\rho$ is the density, $u_{i}$ is the displacement and the components $x_{k}$ are any convenient basis of three orthogonal vectors (Federov 1968, Landau and Lifshitz 1970, Musgrave 1970). The repeated index convention is observed throughout. The stress tensor $\sigma_{i k}$ is in general related to the strain tensor $u_{l m}$ by

$$
\sigma_{i k}=c_{i k l m} u_{l m}
$$

The elastic tensor $c_{i k l m}$ has $3^{4}=81$ elements, but crystal symmetries and stability criteria reduce the number that are independent. For sapphire, there are six independent elastic constants, which are noted in table 1 (Farnell 1961).

Table 1. Elastic constants of sapphire $\left(10^{11} \mathrm{~N} \mathrm{~m}^{-2}\right.$ : Farnell 1961).

\begin{tabular}{lccccc}
\hline$c_{11}$ & $c_{33}$ & $c_{43}$ & $c_{12}$ & $c_{13}$ & $c_{14}$ \\
\hline 4.968 & 4.981 & 1.474 & 1.636 & 1.109 & -0.235 \\
\hline
\end{tabular}

These $c_{m n}$ form a $6 \times 6$ matrix, the remainder of whose elements are given by $c_{22}=c_{11}$, $c_{23}=c_{12}, c_{55}=c_{44},-c_{24}=c_{56}=c_{14}, c_{66}=\frac{1}{2}\left(c_{11}-c_{12}\right)$. All others are equal to zero. The elastic tensor $c_{i j k l}$ may be constructed from these elements by replacing subscripts, $m \rightarrow i, j$ and $n \rightarrow k, l$ according to the following scheme: $1 \rightarrow 1,1 ; 2 \rightarrow 2,2 ; 3 \rightarrow 3,3 ; 4 \rightarrow$ $2,3=3,2 ; 5 \rightarrow 1,3 ; 6 \rightarrow 2,1$. 
In terms of the elastic tensor, Newton's law takes the form

$$
\rho\left(\partial^{2} u_{i} / \partial t^{2}\right)=c_{i j l m}\left(\partial^{2} u_{m} / \partial x_{j} \partial x_{l}\right)
$$

Substitution into equation (3) of plane-wave displacements

$$
u_{i}=e_{i} \exp [\mathrm{i}(\boldsymbol{k}, \boldsymbol{x}-\omega t)
$$

where $\hat{e}$ is a unit polarisation vector and $\boldsymbol{k}$ and $\omega$ are the wavevector and frequency yields the eigenvalue problem

$$
\left(\lambda_{i j l m} n_{l} n_{j}-v^{2} \delta_{i m}\right) e_{m}=0 .
$$

Here $\lambda_{i j l m}=(1 / \rho) c_{i j l m}$ and $\boldsymbol{k}=(\omega / v) \hat{n}$ where $\hat{n}$ is a unit vector.

The solutions of equation (4) determine the three orthogonal polarisation vectors and phase velocities $v$ for any given direction $\hat{n}$, but the polarisations have no particular orientation with respect to $\hat{n}$ or the surface of the crystal, so they can only be described as quasilongitudinal or quasitransverse. The directions along which energy is transmitted are given by the power-flow vector $p$ :

$$
p_{i}=-\sigma_{i k}\left(\partial u_{k} / \partial t\right) \text {. }
$$

The time-averaged power flow may be written in terms of the elastic tensor as

$$
\bar{p}_{i}=\frac{1}{2} \omega c_{i j l m} k_{l} e_{j} e_{m}
$$

Thus, an elastic wave in an anisotropic medium is described by three vectors $\boldsymbol{k}, \hat{e}$ and $\boldsymbol{p}$ whose mutual orientation is a complicated function of direction.

As in an isotropic solid, translational invariance along the surface requires that on reflection, the component of $\boldsymbol{k}$ parallel to the surface must be conserved. However, this law cannot be expressed in simple trigonometric form because the magnitude of $k$ for each polarisation depends on the direction in the crystal. The values of the reflected wavevectors for any given incident geometry must generally be found by solving a sixth-order polynomial equation, the solutions of which may be represented graphically as shown in figure 4. Here, in a polar plot, curves of constant $|\boldsymbol{k}|$ for each mode in the plane of incidence are drawn. The intersections of those clirves with the line given by $k_{\|}^{\text {ref }}=k_{\|}^{\text {in }}$ (the symbols represent the parallel components of the reflected and incident

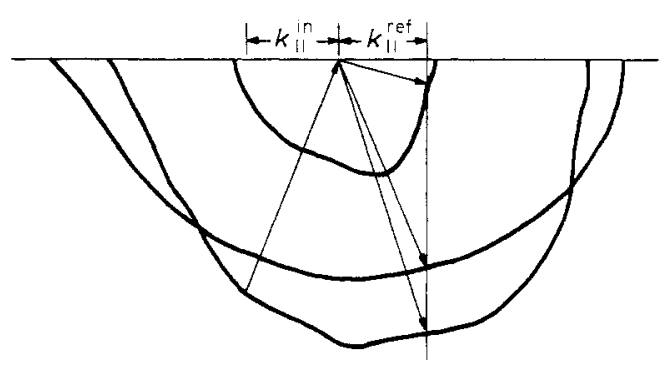

Figure 4. Schematic polar plot of curves of constant $|k|$ (with $\omega$ a fixed constant) in a particular crystallographic plane. The inner curve represents the quasilongitudinal mode, which always has the largest phase velocity. The larger curves correspond to the two quasitransverse modes. The $k$ vectors of the three reflected waves, which are related to the incident wave by the relation $k_{\|}^{\text {in }}=k_{\mid !}^{\text {ref }}$, are determined by the geometrical construction shown in the figure. For sufficiently large $k$, the construction may yield less than three intersections; this corresponds to complex $k$-vectors which represent exponentially damped waves. 
wavevectors respectively) give the permitted solutions for outgoing waves. The line may intersect all three polarisations, giving rise to three outgoing waves, or there may be evanescent solutions as occur in an isotropic medium when the critical angle is exceeded.

The curves shown in figure 4 are described algebraically by the condition

$$
\operatorname{det}\left(\lambda_{i j l m} k_{l} k_{j}-\delta_{i m}\right)=0
$$

which yields a very complicated polynomial in the components of $\boldsymbol{k}$. It is easier to evaluate the coefficients of the polynomial in a rotated frame in which $k_{Y}^{\text {in }}=0$. In this frame $\boldsymbol{k}^{\mathrm{in}}=\left(k_{\|}^{\mathrm{in}}, 0, k_{\perp}^{\mathrm{in}}\right)$ and $\boldsymbol{k}^{\mathrm{ref}}=\left(k_{\|,}^{\mathrm{in}}, 0, k_{\| !}^{\text {ref }}\right)$. Since $k_{\|}^{\text {in }}$ is given, equation (7) reduces to a sixth-order polynomial equation in the single variable $k_{\perp}^{\text {ref }}$. Three of the solutions (those with $\operatorname{Re}\left(k_{i !}^{\text {ref }}\right)>0$ ) correspond to refracted modes which are not allowed at a vacuum interface. Solutions with $\operatorname{Re}\left(k_{\mid:}^{\text {ref }}\right)<0$ correspond to possible reflection processes, although if $k_{\perp}^{\text {ref }}$ has a complex part, the reflected wave will be exponentially damped.

Once the permitted solutions have been determined, the amplitude of each is obtained using (as in the isotropic system) the stress-free boundary condition at the surface:

$$
\sigma_{i k} m_{k}=0
$$

where $\hat{m}$ is the surface normal.

If we imagine a coordinate frame defined by the polarisation vectors for each $\boldsymbol{k}$, then the coupling between the various modes upon reflection is proportional to the projection of a given incident polarisation on each of the three reflected polarisations. In other words, the coupling is governed by how much the coordinate frame twists when the waves change direction because of reflection. The important point here is that the twisting of the polarisation frame is not simply related to the anisotropy of the phase velocity. That is why, even for sapphire, which is almost isotropic, modemixing in an arbitrary orientation may be strong, giving rise to complicated results like those shown in figure 2.

Computation of the trajectories that transport energy from the heater to the detector is particularly difficult because energy flows along the $p$ vector, but reflection is governed by the $\boldsymbol{k}$ vector which is not collinear with it. Moreover, the vector $\boldsymbol{k}^{\text {in }}$ of an emitted beam that will result in a signal at the detector need not be in the plane that contains the heater, detector and surface normal. It is not uncommon to find the necessary $\boldsymbol{k}^{\text {in }}$ more than $30^{\circ}$ out of that plane. Thus the process of finding the right path for a given combination of modes is similar to artillery ranging. A beam is sent in some direction from the heater and the point at which energy in the desired reflected mode arrives back at the same surface is computed. The aiming direction is then adjusted and the computation repeated until a hit is scored on the bolometer. To complicate matters further, the time of flight of (say) the $S V \rightarrow L$ path is no longer equal to that of the $L \rightarrow S v$ path because, unlike an isotropic solid, each of the velocities now depends on direction. Instead of the three processes shown in the inset of figure 3, an anisotropic solid has nine distinct channels that connect the heater and the bolometer and the above artillery practice must be repeated nine separate times for each configuration of the heater and bolometer to predict a complete spectrum.

If the incident (i.e. emitted) and reflected $p$ vectors are, respectively, $\boldsymbol{p}^{\text {in }}$ and $\boldsymbol{p}^{\mathrm{ref}}$, the mathematical criterion for scoring a hit on the bolometer is

$$
\left.H\left\{\left[\boldsymbol{p}_{\text {in }} /\left(\boldsymbol{p}_{\text {in }}, \hat{m}\right)\right]+\boldsymbol{p}_{\text {ref }} /\left(\boldsymbol{p}_{\text {ref }} \cdot \hat{m}\right)\right]\right\}=\boldsymbol{x}
$$


where $H$ is the thickness of the crystal and $x$ is the vector separation of the heater and the bolometer. A computer is programmed to guess $\boldsymbol{k}^{\text {in }}$, to calculate $\boldsymbol{k}^{\text {ref }}$ by imposing the condition $k_{\|}^{\text {in }}=k_{\|}^{\text {ref }}$ and to solve numerically, using equation (7), for the intersections shown in figure 4. Equations (4) and (6) are then used to find the associated $p$ vectors for each combination of polarisations. If $\boldsymbol{p}^{\text {in }}$ and $\boldsymbol{p}^{\text {ref }}$ do not satisfy equation (9) to within a tolerance set by the size of the generator and detector, $\boldsymbol{k}^{\text {in }}$ is corrected and the calculation repeated until the error is satisfactorily small. Moreover, the calculation must be repeated until all nine channels have been found.

Prediction of the received spectrum in detail, including pulse shapes, heights and widths, is a very much more complicated problem that will be discussed briefly in $\S 4$. Our purpose here is principally to compare experimentally observed times of flight for the various channels with the results computed as outlined above. We turn to that comparison in the next section.

\section{Experimental results}

To test the calculations outlined in the previous section, we have performed experiments using a crystal, orientation of which was chosen to simplify the spectrum and facilitate the computations in at least one plane of incidence. The crystal was cylindrical, $9.53 \mathrm{~mm}$ thick and with the two faces (which are optically polished; estimated roughness scale $\approx 30 \mathrm{~nm}$ ) lying in the $C-X$ plane of the sapphire lattice (the $X, Y$ and $C$ axes in the sapphire lattice are shown in figure 5). Thus the surface normal is always the $Y$ axis. Alternative standard designations are $90^{\circ} \mathrm{M}$ or $\langle 1 \overline{1} 00\rangle$ normal to the faces. The diameter of the cylinder, $57.2 \mathrm{~mm}$, was large enough to ensure that reflections from the side walls did not interfere with the spectrum.

The $C-Y$ plane is a particularly simple one for phonon propagation in sapphire because one of the transverse modes had polarisation perpendicular to the plane and the other two polarisations are contained in it. Moreover, for waves propagating in the $C-Y$ plane, the $k$ and $p$ vectors, while still not collinear, are at least all in the plane, which greatly reduces the number of iterations needed for the calculations (the coplanar feature is especially economical since each change in the $\boldsymbol{k}^{\text {in }}$ surface-normal plane re-

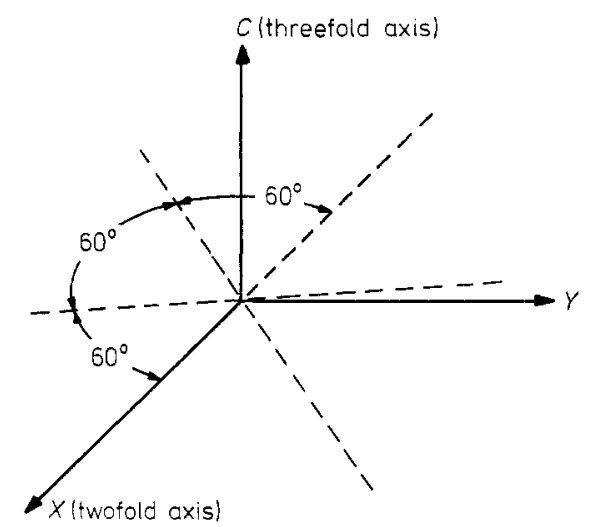

Figure 5. The $X-Y-C$ coordinate system. The $C$ axis is the body diagonal of the trigonal unit cell of sapphire. There are three equivalent twofold axes in the plane perpendicular to $C$, one of which is chosen to be the $X$ axis. The $Y$ axis is then chosen perpendicular to the $C-Y$ plane to form a right-handed system as shown. 
quires rewriting the elastic tensor in a new basis). As in an isotropic solid, the mode with polarisation perpendicular to the $C-Y$ plane is decoupled from the other two modes, so that there are only five reflection channels (rather than nine) in this plane. Because of the simple orientation of the polarisation vectors, each mode can be uniquely labelled by its polarisation as L, SV or SH.

We have thus conducted experiments in which the heater, bolometer and surfacenormal lay in the $\mathrm{C}-Y$ plane. For contrast, we also present results with the heaterbolometer-surface-normal in the $X-Y$ plane, where none of the above simplifications are applicable. In the first case, one bolometer and five heaters were laid out at intervals along the $C$ axis. In the second case a bolometer and five heaters were laid out along the $X$ axis. In addition to testing our calculations, the data presented here constitute, to our knowledge, the first systematic study of phonon reflection as a function of angle of incidence (see however Long et al 1974).

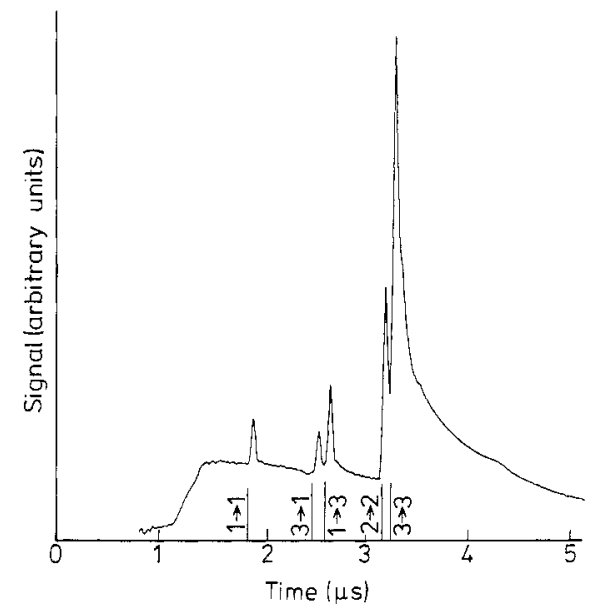

Figure 6. Spectrum from heater number 3 in the $C-Y$ plane (see table 3 for details). Predicted arrival times of the various channels are marked on the abcissa.

An example of a spectrum on the $C-Y$ plane is shown in figure 6 . Here the heater (number 3) and bolometer are separated by $6.7 \mathrm{~mm}$ along the $C$ axis. Five peaks can be seen, just as expected. Figure 2, shown above, was taken in the same crystal with the heater and bolometer separated by $9.0 \mathrm{~mm}$ along the $X$ axis.

Because the purpose of these experiments was to measure times of flight for as many channels as possible in each orientation, all experimental parameters were chosen to optimise the resolution of peaks such as those seen in figures 2 and 7 . The width of a peak is a time that is a consequence of some six separate phenomena, each of which may in turn be characterised by a time: the width of the initial heater pulse, the thermal relaxation times of the heater and the bolometer, effective times due to solid-angle effects at the heater and bolometer and the aperture of the boxcar integrator used to read out the data.

Heater pulses $35 \mathrm{~ns}$ wide were used. Since the signal was observed to scale linearly with the square of the heater pulse height in the range $3-10 \mathrm{~V}$, the pulse height could be chosen within this range to optimise the accuracy of the read-out electronics. The relaxation times of the heater and bolometer were not known accurately, but were minimised by being at an interface between sapphire and superfluid helium. They are believed to be less than $50 \mathrm{~ns}$. Broadening arising from the solid angle subtended by the heater and the bolometer may be estimated from $(w / v) \sin \theta$ where $w$ is a dimension of 
the device, $v$ is the speed of sound and $\theta$ is the angle of incidence or reflection. The heaters were of serpentine form localised to $0.4 \mathrm{~mm}^{2}$ (with resistance $R \approx 40 \Omega$ at $T=2 \mathrm{~K}$ ); the bolometer was slightly larger. Since in sapphire $v \approx 10^{7} \mathrm{~mm} \mathrm{~s}^{-1}$, we estimate a characteristic time of up to $\sim 50 \mathrm{~ns}$ for these geometric effects. Finally, the boxcar aperture was set at $25 \mathrm{~ns}$. Thus, all of these times were of the same order of magnitude, around $50 \mathrm{~ns}$. The times interact in a complicated way, but the resulting peaks may be observed to have widths of roughly $50 \mathrm{~ns}$. It is probably possible to resolve peaks if they are more than $\sim 25 \mathrm{~ns}$ apart. The absolute time of flight of each mode, measured from the beginning of the heater pulse (detected on the bolometer signal by electronic cross-

Table 2. Heater-bolometer-surface-normal in the $C-Y$ plane.

\begin{tabular}{|c|c|c|c|c|c|}
\hline & Channel & $\hat{e}_{\mathrm{in}} \cdot \hat{e}_{\mathrm{ref}}$ & $\theta_{\mathrm{in}}(\mathrm{deg})$ & $t(\mu s)$ predicted & $t(\mu s)$ observed \\
\hline \multicolumn{6}{|c|}{ Heater number $1:$ heater-bolometer separation $=1.5 \mathrm{~mm} ; 0_{0}=4.57^{\circ}$} \\
\hline $1 \rightarrow 1$ & $\mathrm{~L} \rightarrow \mathrm{L}$ & 0.987 & $7 \cdot 45$ & 1.714 & $1 \cdot 71$ \\
\hline $3 \rightarrow 1$ & $\mathrm{SV} \rightarrow \mathrm{L}$ & 0.0205 & 0.57 & $2 \cdot 428$ & $2 \cdot 43$ \\
\hline $1 \rightarrow 3$ & $\mathrm{~L} \rightarrow \mathrm{SV}$ & 0.206 & $10 \cdot 3$ & $2 \cdot 455$ & \\
\hline $2 \rightarrow 2$ & $\mathrm{SH} \rightarrow \mathrm{SH}$ & 10 & 4.57 & 2.956 & $2 \cdot 93$ \\
\hline $3 \rightarrow 3$ & $\mathrm{SV} \rightarrow \mathrm{SV}$ & 0.998 & $2 \cdot 29$ & $3 \cdot 156$ & $3 \cdot 10$ \\
\hline \multicolumn{6}{|c|}{ Heater number 2: heater-bolometer separation $=4.3 \mathrm{~mm} ; \theta_{0}=12.8^{\circ}$} \\
\hline $1 \rightarrow 1$ & $L \rightarrow L$ & 0.89 & $17 \cdot 18$ & $1 \cdot 765$ & 1.74 \\
\hline $3 \rightarrow 1$ & $\mathrm{SV} \rightarrow \mathrm{L}$ & 0.147 & $4 \cdot 18$ & $2 \cdot 442$ & $2 \cdot 42$ \\
\hline $1 \rightarrow 3$ & $\mathrm{~L} \rightarrow \mathrm{SV}$ & $0 \cdot 385$ & $18 \cdot 5$ & 2.523 & $2 \cdot 49$ \\
\hline $2 \rightarrow 2$ & $\mathrm{SH} \rightarrow \mathrm{SH}$ & $1 \cdot 0$ & $16 \cdot 0$ & 3.044 & $2 \cdot 98$ \\
\hline $3 \rightarrow 3$ & $\mathrm{sV} \rightarrow \mathrm{sV}$ & 0.987 & $6 \cdot 3$ & $3 \cdot 191$ & $3 \cdot 14$ \\
\hline \multicolumn{6}{|c|}{ Heater number $3:$ heater-bolometer separation $=6.7 \mathrm{~mm} ; \theta_{0}=19.5^{\circ}$} \\
\hline $1 \rightarrow 1$ & $L \rightarrow L$ & 0.748 & $24 \cdot 6$ & 1.851 & 1.84 \\
\hline $3 \rightarrow 1$ & $\mathrm{SV} \rightarrow \mathrm{L}$ & 0.302 & $8 \cdot 59$ & $2 \cdot 484$ & $2 \cdot 48$ \\
\hline $1 \rightarrow 3$ & $1 \rightarrow S V$ & $0 \cdot 516$ & $23 \cdot 5$ & $2 \cdot 602$ & $2 \cdot 58$ \\
\hline $2 \rightarrow 2$ & $\mathrm{SH} \rightarrow \mathrm{SH}$ & $1 \cdot 0$ & $24 \cdot 1$ & $3 \cdot 165$ & $3 \cdot 11$ \\
\hline $3 \rightarrow 3$ & $s v \rightarrow s v$ & 0.97 & $9 \cdot 62$ & 3241 & $3 \cdot 20$ \\
\hline \multicolumn{6}{|c|}{ Heater number $4:$ heater-bolometer separation $=9.3 \mathrm{~mm} ; \theta_{0}=25.9^{\circ}$} \\
\hline $1 \rightarrow 1$ & $\mathrm{~L} \rightarrow \mathrm{L}$ & 0.57 & $30 \cdot 4$ & 1.967 & 1.94 \\
\hline $3 \rightarrow 1$ & $S V \rightarrow L$ & 0.479 & $13 \cdot 6$ & 2.566 & 2.53 \\
\hline $1 \rightarrow 3$ & $\mathrm{~L} \rightarrow \mathrm{SV}$ & 0.65 & $29 \cdot 6$ & 2718 & $2 \cdot 68$ \\
\hline $2 \rightarrow 2$ & $\mathrm{SH} \rightarrow \mathrm{SH}$ & 1.0 & $31 \cdot 0$ & $3 \cdot 318$ & 3.26 \\
\hline $3 \rightarrow 3$ & $\mathrm{sv} \rightarrow \mathrm{sV}$ & 0.942 & $13 \cdot 4$ & $3 \cdot 320$ & 520 \\
\hline \multicolumn{6}{|c|}{ Heater number $5:$ heater-bolometer separation $=12.6 \mathrm{~mm} ; \theta_{0}=33.4^{\circ}$} \\
\hline $1 \rightarrow 1$ & $\mathrm{~L} \rightarrow \mathrm{L}$ & 0.36 & $35 \cdot 6$ & $2 \cdot 134$ & $2 \cdot 12$ \\
\hline $3 \rightarrow 1$ & $\mathrm{SV} \rightarrow \mathrm{L}$ & 0.702 & $20 \cdot 0$ & $2 \cdot 710$ & $2 \cdot 69$ \\
\hline $1 \rightarrow 3$ & $\mathrm{~L} \rightarrow \mathrm{SV}$ & 0.788 & $35 \cdot 5$ & 2896 & 287 \\
\hline $3 \rightarrow 3$ & $\mathrm{SV} \rightarrow \mathrm{SV}$ & 0.87 & $19 \cdot 2$ & $3 \cdot 471$ & 3.45 \\
\hline $2 \rightarrow 2$ & $\mathrm{SH} \rightarrow \mathrm{SH}$ & 1.0 & $40 \cdot 4$ & 3.612 & $3 \cdot 55$ \\
\hline
\end{tabular}

talk) and the beginning of the rise of the peak is probably determined to better than $50 \mathrm{~ns}$, but there is a geometric uncertainty of that order inherent in the iterative process used to predict the times of flight.

The results of calculation and experiment for the heater-bolometer-surface-normal in the $C-Y$ plane are summarised in table 2 ; the spectra themselves are shown in figure 7. Data and calculations for the $X-Y$ plane are given in table 3 .

Each of these tables has a separate section for each heater, headed by the heaterbolometer separation and $\theta_{0}$, the angle of incidence for which an equal angle of reflection would connect the heater and bolometer. The first two columns in table 2 give the channel 
that connects the heater and bolometer. The channels for each heater are listed in order of their predicted arrival time. The modes within each channel are identified first by number, 1,2 and 3 , then by name, $\mathrm{L}$, SH and sv respectively. Identification by name is possible only in the $C-Y$ plane; even here it would be more accurate to say quasi-L and quasi-sv. The number scheme (also used in the $X-Y$ plane) labels the modes in order of their phase velocities in the direction of the surface-normal. A mode in any direction $\hat{n}$ can be identified with one in the surface-normal direction $\hat{m}$ by tracing along smooth curves like those shown in figure 4 ; the correct curve can be identified by a smoothly varying polarisation. We found that this was the best procedure for monitoring the modes under all possible circumstances. Note, however, that although mode 2 (SH) is the second fastest along the surface-normal, the $\mathrm{SH}-\mathrm{SH}$ channel is the last to arrive when fired from heater number 5 .

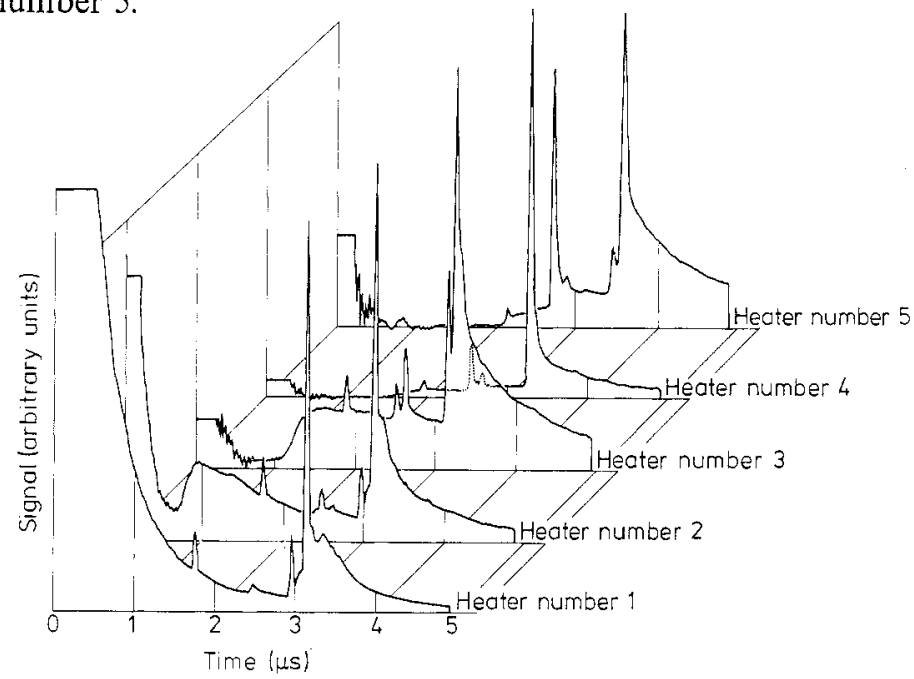

Figure 7. All five spectra in the $C-Y$ plane. The ordinate scales have been chosen so that the tallest peaks in each spectrum are roughly the same height.

The third collimn of table 2 gives the projection of the incident polarisation vector onto the reflected polarisation vector for each channel. This quantity measures the strength of the coupling on reflection from one mode to the other. The channels not listed (i.e. $\mathrm{L} \rightarrow \mathrm{SH}$ ) would have zeros in this column and hence are not expected to arrive. The fourth column of table 2 gives the angle of incidence in the path used by that channel to connect the heater and bolometer. For orientation it may be compared to $\theta_{0}$ which would be the angle of incidence of an $\mathrm{L} \rightarrow \mathrm{L}$ or $\mathrm{T} \rightarrow \mathrm{T}$ channel if the crystal were isotropic. Finally, columns 5 and 6 show the predicted and observed times of flight. The observed times are listed on the same level as the predicted times to which they are believed to correspond.

Table 3 differs from table 2 in that the modes can be identified by number only. In addition, since the required incident $k$-vector does not stay in the $X-Y$ plane, an azimuthal angle $\phi_{\text {in }}$ for the incident (i.e. emitted) ray is also given. The coordinate system that identifies $\theta$ and $\phi$ is shown in figure 8 . In table 3 there are nine channels with nonzero coupling for each heater, all of them listed.

In total, for the ten heaters in both planes, 54 peaks with non-zero coupling and at least $\sim 25$ ns separation are predicted to occur. Every one is observed experimentally, with an arrival time that agrees with the predicted value to within the estimated expected 
Table 3. Heater-bolometer-surface-normal in the $X-Y$ plane

\begin{tabular}{llllll}
\hline Channel & $\hat{e}_{\text {in }}, \hat{e}_{\text {ref }}$ & $\begin{array}{l}\theta_{\text {in }} \\
(\mathrm{deg})\end{array}$ & $\begin{array}{l}\phi_{\text {in }} \\
(\mathrm{deg})\end{array}$ & $\begin{array}{l}t(\mu \mathrm{s}) \\
\text { predicted }\end{array}$ & $\begin{array}{l}t(\mu \mathrm{s}) \\
\text { observed }\end{array}$ \\
\hline
\end{tabular}

Heater number 1 : heater-bolometer separation $=1.5 \mathrm{~mm} ; \theta_{0}=4.50^{\circ}$

$\begin{array}{lccccc}1 \rightarrow 1 & 0.987 & 4.50 & 0.0 & 1.701 & 1.72 \\ 1 \rightarrow 2 & 0.069 & 10.6 & 71.6 & 2.347 & \\ 2 \rightarrow 1 & 0.069 & 6.59 & -72.2 & 2.350 & \\ 1 \rightarrow 3 & 0.177 & 10.89 & 31.5 & 2.448 & \\ 3 \rightarrow 1 & 0.178 & 6.02 & -29.22 & 2.448 & 2.45 \\ 2 \rightarrow 2 & 0.975 & 1.89 & 0.0 & 2.952 & 2.92 \\ 2 \rightarrow 3 & 0.475 & 4.87 & 0.0 & 3.060 & 3.02 \\ 3 \rightarrow 2 & 0.475 & 4.58 & 0.0 & 3.060 & 3.17 \\ 3 \rightarrow 3 & 0.606 & 12.9 & 0.0 & 3.204 & \end{array}$

Heater number 2 : heater-bolometer separation $=4.2 \mathrm{~mm} ; \theta_{0}=12.4^{\circ}$

$\begin{array}{lccrrr}1 \rightarrow 1 & 0.910 & 12.4 & 0.0 & 1.744 & 1.75 \\ 1 \rightarrow 2 & 0.212 & 14.0 & 42.4 & 2.378 & 2.37 \\ 2 \rightarrow 1 & 0.209 & 8.25 & -43.5 & 2.379 & \\ 1 \rightarrow 3 & 0.318 & 21.2 & 12.0 & 2.513 & 2.49 \\ 3 \rightarrow 1 & 0.329 & 11.2 & -13.2 & 2.516 & 2.95 \\ 2 \rightarrow 2 & 0.819 & 5.73 & 0.0 & 2.978 & 3.08 \\ 2 \rightarrow 3 & 0.766 & 13.2 & 0.0 & 3.121 & 3.26 \\ 3 \rightarrow 2 & 0.769 & 12.0 & 0.0 & 3.124 & \\ 3 \rightarrow 3 & 0.525 & 18.9 & 0.0 & 3.331 & 3.26\end{array}$

Heater number $3:$ heater-bolometer separation $=6.6 \mathrm{~mm} ; \theta_{0}=19.3^{\circ}$

$\begin{array}{lrrrrr}1 \rightarrow 1 & 0.788 & 19.3 & 0.0 & 1.803 & 1.80 \\ 1 \rightarrow 2 & 0.359 & 18.9 & 22.9 & 2.425 & 2.38 \\ 2 \rightarrow 1 & 0.361 & 11.2 & -23.6 & 2.429 & \\ 3 \rightarrow 1 & 0.406 & 14.8 & -5.15 & 2.602 & 2.58 \\ 1 \rightarrow 3 & 0.417 & 29.2 & 6.30 & 2.606 & 3.02 \\ 2 \rightarrow 2 & 0.563 & 11.5 & 0.0 & 3.034 & 3.20 \\ 2 \rightarrow 3 & 0.787 & 21.2 & 0.0 & 3.227 & 3.229 \\ 3 \rightarrow 2 & 0.786 & 18.0 & 0.0 & 3.45 \\ 3 \rightarrow 3 & 0.510 & 23.5 & 0.0 & 3.489 & 3.45\end{array}$

Heater number $4:$ heater-bolometer separation $=9.0 \mathrm{~mm} ; \theta_{0}=25.3^{\circ}$

$\begin{array}{llllll}1 \rightarrow 1 & 0.636 & 25.3 & 0.0 & 1.886 & 1.903 \\ 2 \rightarrow 1 & 0.512 & 15.4 & -12.0 & 2.507 & 2.51 \\ 1 \rightarrow 2 & 0.520 & 26.9 & 11.5 & 2.513 & \\ 1 \rightarrow 3 & 0.482 & 36.3 & 2.64 & 2.721 & 2.70 \\ 3 \rightarrow 1 & 0.480 & 18.0 & -2.29 & 2.721 & 3.14 \\ 2 \rightarrow 2 & 0.286 & 20.0 & 0.0 & 3.129 & \\ 3 \rightarrow 2 & 0.723 & 24.4 & -1.26 & 3.381 & 3.35 \\ 2 \rightarrow 3 & 0.721 & 29.2 & 1.14 & 3.388 & 3.57 \\ 3 \rightarrow 3 & 0.510 & 27.2 & 0.0 & 3.665 & 37\end{array}$

Heater number 5 : heater-bolometer separation $=12.7 \mathrm{~mm} ; \theta_{0}=33.7^{\circ}$

$\begin{array}{llllll}1 \rightarrow 1 & 0.383 & 33.7 & 0.0 & 2.054 & 2.05 \\ 1 \rightarrow 2 & 0.717 & 39.5 & 0.0 & 2.689 & 2.67 \\ 2 \rightarrow 1 & 0.718 & 22.6 & 0.0 & 2.691 & \\ 1 \rightarrow 3 & 0.542 & 45.8 & -0.86 & 2.939 & 2.90 \\ 3 \rightarrow 1 & 0.543 & 21.9 & 0.92 & 2.944 & \\ 2 \rightarrow 2 & 0.145 & 37.8 & 0.0 & 3.391 & 3.37 \\ 2 \rightarrow 3 & 0.595 & 38.3 & 1.15 & 3.684 & 3.63 \\ 3 \rightarrow 2 & 0.593 & 32.1 & -1.15 & 3.688 & \\ 3 \rightarrow 3 & 0.549 & 32.1 & 0.0 & 3.974 & 3.91\end{array}$


error. We thus believe that the calculations and procedures we have described have been fully validated.

These results have a number of interesting features that should be noted. For four out of the five heaters in the $C-Y$ plane, the SV $\rightarrow \mathrm{L}$ and $\mathrm{L} \rightarrow \mathrm{sv}$ channels are clearly resolved. One result is that in three of the cases five peaks, which is the maximum number possible, are clearly seen. By contrast, in the $X-Y$ plane, all of the mode-conversion peaks arrive within 10 ns of their inverse channels. For example, in heater number 2 in the $X-Y$ plane, the $1 \rightarrow 3$ and $3 \rightarrow 1$ channels are expected at 2.513 and $2.516 \mu$ s respectively, although

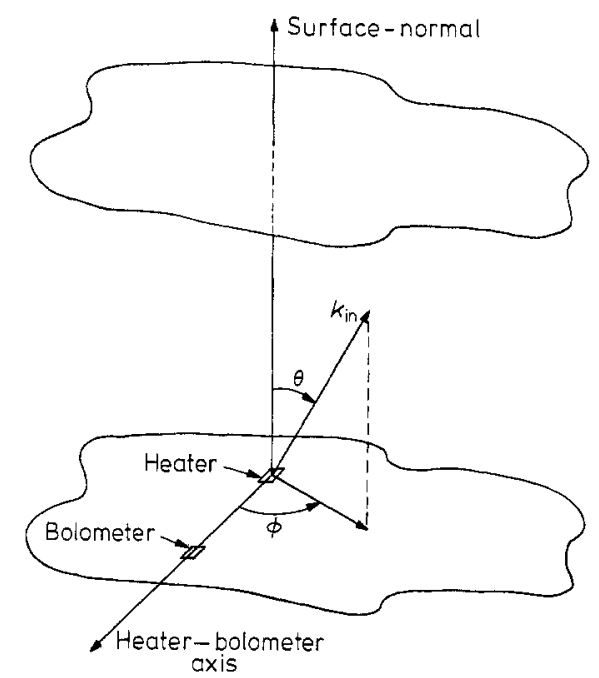

Figure 8. Coordinate system used to define $\theta$ and $\phi$, as given in tables 2 and 3 .

they must have followed quite different paths through the crystal. The experimental consequence is that it is never possible to resolve more than six separate peaks in this plane (as seen, e.g., in figure 2). This behaviour is apparently a geometrical accident that is peculiar to the $X-Y$ plane. As mentioned above, in previous experiments on a crystal whose orientation was not well known, we observed as many as eight peaks. Calculations for propagation in arbitrarily chosen crystal orientations indicate that seven or eight peaks should often be resolved by 20 ns or more.

So far, little has been said about the relative heights of the peaks in the various channels. Only a small amount of progress can be made on this point without elaborate new calculations, but a brief discussion may be useful, in order to bring out where the complexities lie.

Let us leave aside for the present the difficult question of what distribution of phonons actually emerges from the heater into the crystal. Some progress can still be made by the following argument. The L-L peak height (for example) is proportional to the intensity of the $\mathrm{L}$ beam incident at the surface, multiplied by the $\mathrm{L}-\mathrm{L}$ reflection efficiency at the interface, given by $\hat{e}_{\text {in }}$. $\hat{e}_{\text {ref }}$. The L-SV peak height should be given by the incident $\mathrm{L}$ intensity at the surface multiplied by the L-SV conversion efficiency. Thus, if we denote the peak heights in each channel $S_{i-j}$ and the conversion efficiencies $R_{i-j}$, it might be expected that

$$
S_{\mathrm{L}-\mathrm{SV}}=R_{\mathrm{L}-\mathrm{sv}}\left(S_{\mathrm{L}-\mathrm{L}} / R_{\mathrm{L}-\mathrm{L}}\right)
$$


The same would be true, of course, for any other combination of converted and unconverted channels.

Consider, for example, heater number 1 in the $C-Y$ plane. The SV $\rightarrow \mathrm{L}$ channel and the $\mathrm{L} \rightarrow$ SV channel are expected to arrive $27 \mathrm{~ns}$ apart, at the edge of resolvability. However, the $\mathrm{L} \rightarrow \mathrm{sv}$ coupling is 0.2 whereas the $\mathrm{sV} \rightarrow \mathrm{L}$ coupling is only 0.02 . One small peak is observed at the expected arrival point, which on careful inspection proves to be noticeably broader than the other specular peaks in the same spectrum. We wish to know whether this peak can be assigned to one channel, or whether it is an unresolved combination of both.

Application of the above argument indicates it probably belongs to both. The reason is that although $R_{\mathrm{L}-\mathrm{SV}} \approx 10 R_{\mathrm{SV}-\mathrm{L}}$, we observe $S_{\mathrm{SV}-\mathrm{SV}} \approx 10 S_{\mathrm{L}-\mathrm{L}}$ (see figure 8; the $\mathrm{L}-\mathrm{L}$ and SV-SV peaks are, respectively, the first and last for heater number 1 ). Thus we expect $S_{\mathrm{L}-\mathrm{SV}} \approx S_{\mathrm{SV}-\mathrm{L}}$. Quantiatively the argument predicts that each channel will contribute a peak, the height of which is about $\frac{3}{4}$ the maximum of the observed peak. Thus the argument agrees very well with observation if the peak is an unresolved doublet. If the channels were of greater intensity, we could probably resolve the two peaks.

After systematic application of the same analysis to all other relevant combinations of peaks, we find reasonable agreement in about half the cases, but serious disagreement in at least some instances. For example, for $C-Y$ heater number 3 (figure 7), $R_{\mathrm{SV}-\mathrm{L}} / R_{\mathrm{SV}-\mathrm{SV}} \simeq 0.3$. We thus expect that the SV-L peak (the second to arrive) will be about $\frac{1}{2}$ the height of the SV-SV peak (the last one). After subtracting the background, however, we find that the former peak is only about $\frac{1}{10}$ the height of the latter, or about three times smaller than expected.

We believe that such discrepancies arise from the phenomenon of phonon focusing in the incident mode. In the example cited, the SV beam that reflects into the SV-SV channel does not follow the same path as the SV beam that reflects into the $\mathrm{sV} \rightarrow \mathrm{L}$ channel. Even if the heater emits an isotropic distribution of $\mathrm{sv}$ phonons, the two beams will not generally have the same intensity. Thus the argument we have presented needs to be corrected for the effects of phonon focusing in the incident mode. We expect to present the required analysis in future work, including all crystallographic effects.

In most previous experiments reporting phonon reflection spectra, emphasis has generally been laid on changes in the spectra that occur when helium is brought into contact with the reflecting surface. It is our contention that the interpretation of those experiments will necessarily remain contradictory and confusing until details such as those we are describing here are fully understood. We wish now to comment on some features of our observations that seem relevant to the problem of transmission of energy through the interface. A systematic account of the behaviour of our spectra upon admitting helium to the cell will be presented later.

For heaters numbers 1,2 and 3 in the $C-Y$ plane, the sv mode is the slowest and the $\mathrm{SV} \rightarrow \mathrm{SV}$ peak is the last to arrive. However, in heater number 4 , the $\mathrm{sV} \rightarrow \mathrm{sv}$ peak arrives almost in coincidence with the $\mathrm{SH} \rightarrow \mathrm{SH}$ peak ( 3.320 compared with $3.318 \mu \mathrm{s})$. In heater number 5 , the $\mathrm{SH} \rightarrow \mathrm{sH}$ peak stands alone, the last to arrive. This observation shows that it is possible, with a carefully designed geometry, to isolate the $\mathrm{SH} \rightarrow \mathrm{sH}_{\mathrm{H}}$ peak for separate study. According to elastic theory, an SH mode should always suffer total internal reflection at a crystal-liquid interface, just as it does at a crystal-vacuum interface, because it cannot couple to longitudinal modes in the liquid. This simplification in the predicted behaviour might make this channel particularly rewarding for isolation and study.

Finally, we come to a feature that is immediately obvious in figure 2 but has not yet been mentioned. For each of the spectra in the $X-Y$ plane, typified by that shown in 
figure 2, the last predicted channel, always observed as a narrow (i.e. width $\sim 50 \mathrm{~ns}$ ) peak, is followed by a prominent broad bump. A broad bump following the (unresolved) transverse peaks has been noted previously (Folinsbee and Harrison 1978). In our experiments we invariably observed it in the $X-Y$ but not in the $C-Y$ plane. We cannot yet pinpoint the precise cause of this feature, but can say with certainty that it arises from

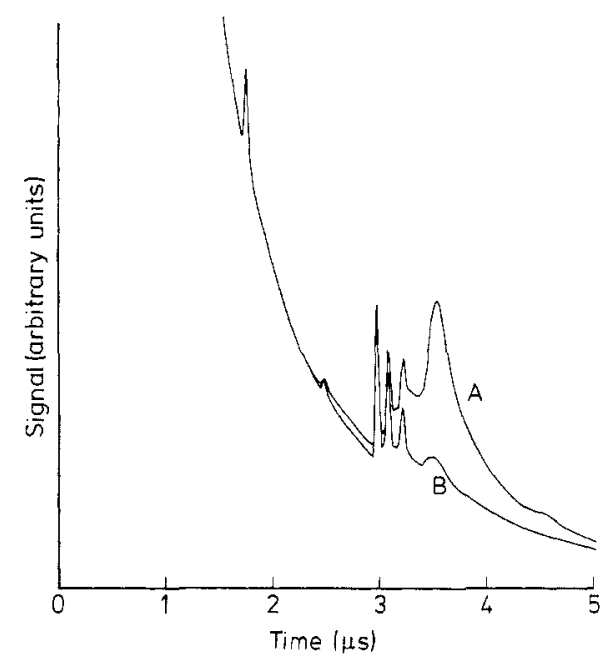

Figure 9. Spectrum from heater number 1 in the $X-Y$ plane (see table 3 for details). Curve $C$, reflecting surface in vacuum; curve $B$, reflecting surface in liquid helium. Notice the effect of helim on the broad bump after the last sharp peak.

phenomena that occur at the surface. This is known because the principal qualitative effect on the $X-Y$ spectra of admitting helium to the reflecting interface is to diminish dramatically the size of the bump, as shown in figure 9.

\section{Conclusions}

Although important insights about surface and interfacial phenomena have been gained from phonon reflection experiments, we believe that the method will not be fully exploited until all of the phenomena governing the detected spectrum are understood. In a sense, the problem is similar to that in other forms of spectroscopy, where the positions of lines are easily understood but predicting their shapes is a more formidable problem. This paper shows how the locations of all of the peaks in the spectrum may be accounted for or predicted. However, the lineshapes, heights and widths as well as other features of the spectra must also be understood before the method can be used for systematic quantitative studies.

The following factors enter into the details of spectra like those presented here:

(i) The width, height and shape of the pulse in the heater.

(ii) The distribution of phonons that emerge.

(iii) Crystallographic effects on transmission (e.g. phonon focusing).

(iv) The times of flight in all channels that connecting the heater and detector in flatsurface reflection. 
(v) The effects of surface roughness.

(vi) The effects of transmission through the surface if there is a medium on the other side (including effects at the heater-crystal and bolometer-crystal interfaces).

(vii) The background signal due, e.g., to the scattering of phonons in the bulk of the crystal;

(viii) Solid-angle affects at the heater and bolometer.

(ix) The thermal response of the bolometer.

(x) The resolution of the read-out instrumentation.

If other methods of generation and detection are used, for example, superconducting tunnel junctions in place of the heater and/or bolometer, details of these factors change, but the basic problems all remain.

It is remarkable that although each of these factors presents difficulties, progress can be made towards understanding every one of them. It is not beyond hope that we will eventually be able to calculate, measure or control, probably everything apart from (vi), which is basically the anomalous Kapitza resistance, and even then we may be able to parametrise this factor so that it can be studied systematically.

In this paper, we have shown how to resolve point (iv) of the list and the same means enable us to deal with point (iii). We believe this to be an essential step towards conquering the overall problem. Further new results, particularly concerning points (v), (vi), and (vii) should soon be ready for publication.

\section{References}

Farnell G W 1961 Can. J. Phys. 39 65-80

Federov F I 1968 Theory of Elastic Waves in Crystals (New York: Plenum)

Folinsbee J T and Harrison J P 1978 J. Low Temp. Phys. 32 469-79

Guo C-J and Maris H J 1972 Phys. Rev. Lett. 29 855-8

Horstman R E and Wolter J 1977 Phys. Lett. 62 A 279-318

Kinder H and Dietsche W 1974 Phys. Rev. Lett. 33 578-81

Landau L D and Lifshitz E M 1970 Theory of Elasticity (New York: Pergamon)

Long A R, Sherlock R A and Wyatt A F 1974 J. Low Temp. Phys. 15 523-36

Maris H J 1970 J. Acoust. Soc. Am. $50812-8$

Musgrave M J P 1970 Crystal Acoustics (San Francisco: Holden-Day)

Taylor B, Maris H J and Elbaum C 1970 Phys. Rev. B 3 1426-72

Weber J, Sandmann W, Dietsche W and Kinder H 1978 Phys. Rev. Lett. 40 1469-71 